\title{
ICOM \\ Investigating the process of ethical approval in citizen science research: the case of Public Health
}

\section{Antonella Ficorilli, Giovanni Maccani, Mara Balestrini, Annibale Biggeri, Bruna De Marchi, Frederique E. M. Froeling, Florence Gignac, Regina Grazuleviciene, Gerard Hoek, Tjaša Kanduč, David Kocman, Valeria Righi and Xavier Basagaña}

\begin{abstract}
Undertaking citizen science research in Public Health involving human subjects poses significant challenges concerning the traditional process of ethical approval. It requires an extension of the ethics of protection of research subjects in order to include the empowerment of citizens as citizen scientists. This paper investigates these challenges and illustrates the ethical framework and the strategies developed within the CitieS-Health project. It also proposes first recommendations generated from the experiences of five citizen science pilot studies in environmental epidemiology within this project.
\end{abstract}

Keywords

Citizen science; Health communication; Participation and science governance

DOI

https://doi.org/10.22323/2.20060204

Submitted: 31st October 2020

Accepted: 11th March 2021

Published: 11th October 2021

Introduction

Undertaking appropriate citizen science research in which citizens act as researchers, i.e. citizen scientists, in Public Health involving human subjects entails significant challenges concerning traditional ethical approval processes. Indeed, current legal rules and ethical requirements focus on protecting the rights and welfare of individuals involved in research projects as human subjects (passive participants) and do not address issues concerning the involvement of individuals as citizen scientists (active participants). These challenges may include aspects regarding: research integrity and information asymmetry deriving from the active involvement of non-professional researchers; trade-offs between the need of a clearly established methodology before ethical approval is granted and the open nature of citizen science interventions. Rather than being fixed in advance, appropriate research methods and techniques emerge over time as a result of co-design and co-creation efforts. This paper proposes an ethical framework as well as some first recommendations generated from the experiences of five citizen 
science pilot studies within the CitieS-Health H2020 project, which has been funded for the three-year period 2019-2021. This framework contributes to advancing the agenda in the direction of extending the current ethics of protection of research subjects towards the inclusion of citizens as citizen scientists. This is done by articulating, distinguishing, and tackling two different roles that citizens may concurrently play in research projects, namely research subjects (traditional role) and citizen scientists (new role), as well as the novelties and challenges that this situation poses on ethical approval processes and on governance. Aspects related to citizens co-creating study protocols are also considered. The environmental epidemiology domain of CitieS-Health and the sensitivity of the research data offer a particularly suitable scenario, which can lead to recommendations for those performing citizen science research in Public Health [Den Broeder et al., 2018].

The project

Co-created projects in Public Health and their ethical aspects
The main objective of the CitieS-Health (https: / / citieshealth.eu/) H2020 project is to develop participatory citizen science pilot studies regarding environmental epidemiology in five European locations. Each pilot study touches on different aspects of urban environmental exposures and their relationship with health, namely air pollution and health in Barcelona (Spain); noise and health in Ljubljana (Slovenia); heavy metal pollution and health in the province of Lucca (Tuscany, Italy); city design and health in Kaunas (Lithuania); and wood burning and health in multiple cities in The Netherlands.

The investigations carried out entail collecting, processing, analysing and using data, and also biological samples in the case of the Italian and Dutch pilots. The data comprises: contact details; health parameters obtained by non-invasive techniques and characteristics that can be related to health, including demographic and socioeconomic data, information on lifestyle/diet, physical activity and occupational history; human biological samples (blood, urine, saliva and mucosal lining fluid of nose); ${ }^{1}$ and environmental data (concentrations of pollutants, noise levels, air quality, urban characteristics of residential place).

The pilots are set up following a participatory governance model carried out by both researchers and citizens, involving the latter at the highest level: i.e. citizens are collaborators who contribute to all phases of the studies together with researchers. This means that the pilots can be qualified as co-created projects [Bonney et al., 2009]. In the case of CitieS-Health, the actors involved in co-creation include researchers belonging to research institutions and universities; small and medium sized enterprises; citizens (both associations and individual citizens); schools (primary and secondary); public authorities (mayors/local administrations); local health units and regional health authorities.

Since the end of World War II, cases of unethical scientific activities have led to the elaboration of specific ethical criteria of research to which every researcher must adhere. These concern honesty and accuracy in collecting, storing, analysing, and reporting data; openness in sharing data, ideas and tools; respect for intellectual property; respect for animals used in research and for the dignity, privacy and autonomy of people enrolled in research studies; social responsibility; transparency

\footnotetext{
${ }^{1}$ In the COVID-19 era specific precautions must be prescribed in the study protocol.
} 
in interaction with the public [National Academy of Sciences, 2009; Resnik and Shampoo, 2009; ALLEA — All European Academies, 2017]. ${ }^{2}$

Consequently, in co-created citizen science projects where citizens act as researchers together with professional researchers, it is necessary that the principles and values of current research ethics become a shared and respected common legacy of rules. Thus, the ethical duty of researchers to act according to ethical standards of good science practice also extends to citizens [Biggeri, 2019; Ficorilli, 2019]. Furthermore, in co-created projects, the sharing of responsibilities between professional researchers and citizen scientists is becoming increasingly evident.

The full participation of citizens implies taking into consideration their active role in all the scientific investigations performed: a) in framing and designing a research study; and b) in implementing a co-designed research study, i.e. performing several scientific activities within the study. As a consequence, a dual active role of citizens emerges, besides their traditional participation as passive subjects of the investigations.

Projects in the field of Public Health and Epidemiology often collect personal and sensitive data, including health parameters and biological samples, and use the principle of pseudonymisation [European Parliament and the Council, 2016]. ${ }^{3}$ Thus, these projects need to address the following three main traditional ethical issues:

1. Respecting the dignity, autonomy and privacy of individuals who provide their personal and sensitive data, including biological samples;

2. Respecting the privacy of individuals whose personal information is obtained - e.g. lifestyle data. Such information may pose issues in terms of surveillance and even discrimination;

3. Ensuring scientific rigor, research integrity and systematic data management in collecting, storing, analysing, interpreting, sharing and using environmental data as well as personal and sensitive data.

Moreover, in such projects, the co-designed study protocols, the related information sheets, and informed consent forms (from now on study protocols and related documents), have to receive approval from a competent Medical Ethics Committee. Such ethics reviews and approval are mandatory as they fall under the research involving human subjects, based on the current European and national normative provisions and guidelines (see section 'Traditional ethical requirements for ethics approval').

In addition, the co-created projects involve some new issues that should be considered when tackling ethical approval:

\footnotetext{
${ }^{2}$ For some relevant normative references see Council of Europe [1997], Council of Europe [2005], Council of Europe [2016] and European Parliament and the Council [2016].

${ }^{3}$ This means that all materials obtained in the framework of the project - questionnaires, information from sensors and so on - are identified through a code, and the name and/or other personal data that might allow the identification of the participant are not indicated.
} 
1. How to address the active role of citizens in designing and implementing the project while respecting the three main ethical aspects identified above;

2. How to harmonize the new role of citizens in the project with the current and traditional procedures of ethics approval of research projects promoted by researchers;

3. How to address possible conflicts among citizens' / researchers' points of view in designing and implementing the study (e.g. conflicts on research questions or on how to share and re-use data).

These all pose challenges for ethics approval. Indeed, in the traditional ethical framework, "individuals have traditionally occupied the role of researcher or subject, but not both at the same time. The unification of these two different roles within the same person poses challenges for investigators and oversight committees because legal rules and ethical guidelines focus on protecting the rights and welfare of human subjects and do not address issues that fall outside this domain" [Resnik, 2019, p. 1]. Instead, the ethics for citizens as citizen scientists requires taking into consideration domains such as study design, data quality, ${ }^{4}$ data sharing and intellectual property, reporting misconduct, authorship, and publication. In other words, domains that are strictly connected to the responsible conduct of professional researchers.

Ethical issues concerning participatory citizen science studies have received attention only recently [Patrick-Lake and Goldsack, 2019; Rasmussen, 2019; Rasmussen and Cooper, 2019; Wiggins and Wilbanks, 2019]. The debate is evolving, and institutional guidelines and rules have not been agreed upon yet.

The CitieS-Health approach to ethical approval
In this context, we will illustrate the ethical framework and its implementation for the ethical approval process envisioned as part of the CitieS-Health project in order to address the above-mentioned challenges. Reflections and first lessons learned will be also provided.

\section{Traditional ethical requirements for ethics approval}

The CitieS-Health studies must elaborate study protocols and related documents that fully comply with the current European and national relevant normative provisions and the requirements for ethics approval, according to their specific research actions [Council of Europe, 1997; Council of Europe, 2005; Council of Europe, 2016; European Parliament and the Council, 2016; European Parliament and the Council, 2018; World Medical Association, 2013]. Citizens are recruited as research subjects who are duly informed about the research, its procedures and goals, and who are asked to sign an informed consent, as well as consent to the processing of their data - and consent to collect, store and use biological samples and associated data in both the Italian and Dutch pilots.

\footnotetext{
${ }^{4}$ About this topic Kosmala et al. [2016], Lukyanenko, Parsons and Wiersma [2016] and United States Environmental Protection Agency [2019].
} 
Specifically, from a traditional point of view, the five pilot studies can be qualified as environmental epidemiology research studies (rather than experimental studies or clinical trials planned to assess new treatments or procedures) involving human subjects, namely research on identifiable personal and sensitive data and human materials. The traditional ethical requirements for the submission of these kinds of research project to a competent Medical Ethics Committee can be summarised in the following nine items:

1. Description of the research study (this is actually very detailed and includes information such as motivation of the rationale of the study, motivation of the study design, detailed description of all methods - including questionnaires, diaries, - power calculation, statistical analysis plan, duration of the study).

2. Information on the subjects that have been invited to participate (which types of subjects will take part in the study and their number, inclusion/exclusion criteria, financial compensation or other benefits offered to participants, etc.).

3. Safety (assessment of risks and benefits) and insurance policy arrangements.

4. A statement by the applicant that individuals will not be involved in the research through pressure or inappropriate induction.

5. Copy of informed consent form and information sheet regarding participation in the study; processing of personal data; and, if necessary, collecting, storing and using biological samples and associated data.

6. If the participant of the study is a minor, the holder of parental responsibility or the legal guardian of the minor must provide consent. In addition, the opinion of the minor should be taken into consideration as an increasingly determining factor in proportion to their age and degree of maturity.

7. Arrangements for processing personal data and respecting the duty of confidentiality and the right to privacy: a) informed consent must be obtained; b) procedures to safeguard the privacy of the study participants must be described in detail; c) an analysis of the ethical issues raised by the processing of personal data involved in the study and an explanation of how these issues will be mitigated in practice must be provided. If necessary, data protection impact assessment (DPIA) in line with Article 35 GDPR [European Parliament and the Council, 2016] and supplementary guidance on DPIAs also have to be provided [European Commission, 2018].

8. Conservation of research results and publication policy.

9. Economic aspects of the study and conflicts of interest.

The following ethical principles underlying these requirements, as recapped by David B. Resnik, are: scientific rigor; social value; risk minimization; reasonableness of risks; respect of autonomy by obtaining informed consent; confidentiality/privacy; equitable selection of subjects; data and safety monitoring; protection of vulnerable subjects; independent review by an Ethics Committee [Resnik, 2019, p. 2]. 
In the light of the new ethical issues mentioned in section 'Co-created projects in Public Health and their ethical aspects', the CitieS-Health project envisioned some original aspects relevant for the elaboration of its study protocols, information sheets and informed consent forms in order to receive ethics approval.

The first one concerns the collaboration between researchers and citizens in the elaboration of study protocols and related documents. Thanks to this collaboration, the structure and purpose of the investigation will have a special relevance from both the scientific and the citizen communities' point of view. Indeed, the purpose of the pilots is to collaboratively investigate the health issues that cause concern for the local population in order to generate scientific evidence possibly relevant at both local and global level. In addition, this collaboration may foster a more organic vision of environmental and health issues in the area by providing a chance to reflect on similar issues. Moreover, the participatory approach provides an opportunity to clearly disclose and discuss the limitations of epidemiological studies and ensure transparency in all phases of the study. It is also of paramount importance that researchers and citizens participate in a joint debate concerning research ethics and requirements for ethical approval of a scientific research protocol, as well as concerning possible conflicts that may arise due to differing citizens' / researchers' points of view.

The second original aspect regards the enrolment of citizens as active participants in a co-designed study, rather than being simply research subjects. After the study protocol has been finalized and approved by a competent Ethics Committee, citizens are asked to actively contribute to the implementation of the studies. This means that they are also enrolled as active participants who are asked to perform some scientific activities. Hence, the rise of two different roles of citizens enrolled in a co-designed citizen science study: as an active participant (new role) and as a research subject (traditional role). Note that the citizens already involved in the co-design phase may also be enrolled in the co-implementation phase. If this is the case, special attention should be paid on issues such as possible bias and robustness of the studies.

The third original aspect concerns the consideration of two new responsibilities. The researcher's responsibility to communicate/share current ethical standards (as stated in Regulation EU 679/2016) to/with citizens who will be involved in the study; and the citizen's responsibility to comply with these standards of research ethics when he/she performs scientific activities.

\section{Two-step strategy for ethics approval}

The CitieS-Health strategy envisioned to address the emergent ethical aspects identified above consists in a two-step process for ethical approval, with separate templates for information sheets and consent forms for each step, both involving the new active role of citizens.

In the first step, citizens, as citizen scientists, are asked to contribute to debating and co-designing the study protocol together with researchers (new role). Note 
that in traditional projects, the specific methods and techniques to be employed are stated before starting the project. Unlike this context, in citizen science projects such as the one described here with co-design and co-creation as its core, the research questions to be addressed and the methodologies to be used emerge from an ongoing collaborative process. As the clear methodological plan is not available at the design stage, it makes sense to separate the design step from the implementation step, and envision a separate ethics approval for the design step, addressing its specific ethical issues.

In the second step, once the methodology has been specified, the study must go through the traditional ethical approval. In this step, the main challenge to be addressed regards some new peculiarities related to the active role of participants in this phase. The next sections describe the new elements that CitieS-Health considered introducing within the study protocol and related information sheet and informed consent form to take this new role of citizens into account.

\section{New elements within the study protocol}

The following new elements to be introduced within the study protocol have been identified.

1. Inclusion of the names of individual citizens who co-designed the study protocol and related documents, as co-proponents together with those of the researchers [Buyx et al., 2017]. Note that it is up to the researchers and citizens to decide together which names are to be included [De Marchi et al., 2017]. The co-proposal of the study will imply a co-responsibility of citizens and researchers, although the Principal Investigator (a researcher, according to current laws) will continue to be the person in charge of the study, with adequate education, training and expertise on scientific methods and data management. Moreover, it is assumed that he/she is aware of rules and guidelines concerning research integrity and ethics. This means that, as a general criterion, active participants will perform scientific activities on behalf and under the responsibilities of researchers. Then the Principal Investigator will maintain the scientific and ethical accountability for the solidity of the study and will be the contact person for the Ethics Committee. At the same time, the move towards sharing responsibility has to be considered as a move towards the establishment of an Extended Peer Community [Funtowicz and Ravetz, 1997].

2. Inclusion of an explanation of the best practices followed by researchers, and citizens if this is the case, to engage people in debating and co-designing the study protocol and related documents. In this regard, as mentioned in section 'Two-step strategy for ethics approval', CitieS-Health researchers envisaged a specific first stage engagement process of participants who are asked to actively contribute to the design of the protocol. This first stage only involves discussion with citizens. Researchers might proceed with the recruitment of participants through an information sheet and an informed consent form specifically prepared for this purpose taking into account the new elements described in sections 'New elements within the information sheet' and 'New elements within the informed consent form'. In addition, other good 
practices for the scientific, ethical and social acceptability of the study protocol were identified [Kocman et al., 2019]:

a) Planning and implementing meetings between researchers and citizens specifically dedicated to standards of the current ethics of research, including regulations and rights related to the use, re-use, storage, and sharing of data. These standards should be discussed and shared with citizens thus becoming a common legacy of the project.

b) Adopting a participatory approach in which possible concerns of citizens and researchers are highlighted and addressed to manage conflicts which may arise due to differing citizens' / researchers' points of view on issues such as research questions to be pursued, intellectual property of findings, as well as on how to share and use those findings. Collaboration does not imply adopting a reductionist attitude, but rather enriching both the researchers' and the citizens' views on knowledge and values.

c) Introducing a modification to the traditional peer review process based on the idea of an extended peer review ${ }^{5}$ in order to achieve a public validation of the co-designed epidemiological study protocol, consolidate agreement on the research objectives and strengthen confidence that the selected procedures will be applied correctly and honestly. Hopefully, this will prevent or help resolve any disputes / conflicts concerning the final results and their health policy implications [De Marchi et al., 2017, in particular regarding the public evaluation of study protocols and health implications of the future possible scenarios emerging from the study results].

3. Planning and implementation of training in research integrity and scientific data management for the citizens enrolled as active participants in the co-designed study. As mentioned in section 'Co-created projects in Public Health and their ethical aspects', it is important that principles and values of research ethics are met by both researchers and citizen scientists. To this end, training, debates and sharing of knowledge on ethical standards become a preliminary and fundamental part of the study. Thus, we emphasize, on the one hand, the responsibility of researchers to share these standards with citizen scientists enrolled in the study; and on the other, the responsibility of the enrolled citizen scientists to comply with these standards.

4. Disclosure of financial and/or non-financial conflicts of interest of citizens [Resnik, Elliott and Miller, 2015] who appear as co-proponents of the study protocol. Moreover, researchers and citizens should co-decide, and indicate within the study protocol, the type of conflicts of interest that people who are enrolled as citizen scientists will have to declare on the informed consent form.

5. Commitment to return individual medical/health results to each participant, if she/he so chooses. Any anomalous results at individual level imply an ethical obligation to assure access to the best health care currently available in each country according to current legislation. This must be done with the utmost respect for the privacy of the individual.

\footnotetext{
${ }^{5}$ The expression is derived from that of "extended peer community" introduced by Funtowicz and Ravetz [1993].
} 
6. Guarantee that the plan of publications will include the dissemination of research issues, final study protocol considered from the point of view of both researchers and citizens, study findings, and the type of citizen collaboration adopted. In addition, identification of the names of researchers and citizens who will appear as authors and/or the collective name that will be used in publishing.

\section{New elements within the information sheet}

Based on the considerations above, the following new elements to be introduced within the information sheet have been also investigated, outlined, and determined.

1. Information concerning the two different roles that participants will play and what these two roles will imply for them, taking into account the CitieS-Health pilots (Table 1).

Table 1. CitieS-Health framework for the possible contribution of participants in the five co-designed pilots as research subjects and active participants.

\begin{tabular}{|c|c|}
\hline As a research subject, a citizen will be asked: & As an active participant, a citizen will be asked: \\
\hline $\begin{array}{l}\text { a. To provide his/her personal and sensit- } \\
\text { ive data necessary to perform the study, } \\
\text { according to decisions that will be taken } \\
\text { by each pilot; } \\
\text { b. To provide some of his/her biolo- } \\
\text { gical materials. Namely, blood and } \\
\text { urine samples in the Lucca study; } \\
\text { saliva samples and mucosal lining fluid } \\
\text { samples from the nose in The Nether- } \\
\text { lands pilot; } \\
\text { c. To share his/her data with the other par- } \\
\text { ticipants and researchers, according de- } \\
\text { cisions that will be taken by each pilot. }\end{array}$ & $\begin{array}{l}\text { a. To participate in collecting and ana- } \\
\text { lysing environmental data and per- } \\
\text { sonal and sensitive data relevant to the } \\
\text { study, disseminating results and propos- } \\
\text { ing public health actions, according to } \\
\text { decisions that will be taken by each pi- } \\
\text { lot; } \\
\text { b. On behalf and under the responsibility } \\
\text { of the study Principal Investigator, to } \\
\text { participate in involving other citizens in } \\
\text { the pilot, by informing and asking them } \\
\text { to sign the consent related to participa- } \\
\text { tion and processing of personal data. }\end{array}$ \\
\hline
\end{tabular}

2. Clarification on the specific nature of the study (participatory and citizen science pilot study); the specific purpose of the study (relevant from both the scientific and the citizen communities' points of view); and the potential benefits of taking part in this kind of the study.

3. Details about authorship and acknowledgement policy. Indeed, citizens who contribute to scientific research should be either named as authors or recognized in the acknowledgments. In the case of minors, the suggestion is to give them appropriate credit through a collective name, e.g. the name of the school involved in the study.

4. Information about an "agreement of collaboration" that participants are asked to sign together with the informed consent form (as illustrated in the next section). 
Finally, one further element to be introduced within the informed consent has been conceived.

An "agreement of collaboration" that citizens are asked to sign. This agreement basically allows researchers to use a traditional ethical instrument and procedure (i.e. informed consent) to incorporate new needs, tasks, and actors in research. By signing it, individuals accept to be actively involved in scientific activities on behalf and under the responsibility of the study Principal Investigator. In addition, considered that this agreement of collaboration allows citizens to perform scientific activities, it also implies that:

a) They are aware of the moral responsibility implied in such an active role, i.e. they perform scientific activities fulfilling the current ethical standards of the scientific research;

b) It is their duty to declare any financial and/or non-financial conflict of interest, if present.

Experiences and challenges
This section provides reflections on some key feedback received by the ethics committees during the first half of the CitieS-Health project together with an outline of those challenges that remain open. Some pilots have yet to submit their protocols to the Medical Ethics Committee due to their specific needs and timeline as well as disruption produced by the emergence of COVID-19 starting at the beginning of 2020. Therefore, the results reported will be fully presented with further data and considerations at the end of the project. Although preliminary, this first feedback gives an important opportunity to enrich the scientific debate on how to supplement the current procedures for ethical approval with the new role of citizens as citizen scientists within the European context.

Key feedback received by Ethics Committees regarding both the first and second phase of the ethical approval process can specifically generate reflection on the current challenges of these complex and multi-faceted processes in citizen science research. First, both the Barcelona and Lucca pilots submitted protocols for the co-design phase (first phase) to the Medical Ethics Committees, and both Committees claimed that the matter was not within their competence as they did fall within biomedical regulations. This shows that this part of the study, which involves more social science oriented, could be done without such approval. Yet, we believe it is a matter to be investigated more in depth by involving other Ethics committees that are not the medical ethics committees that researchers are used to working with. This is what was done for the Lucca pilot. Second, the response of the Research Ethics Committee of the University of Florence (Italy), with respect to the population engagement (first phase), provided approval with the recommendation that individual data be accessible only to researchers and not to lay people, who should have access only to aggregated data. Note that this response is of particular interest considering that some citizens were indicated as authors of the protocol together with researchers. In addition, the kind of Committee concerned is also important, namely a University Research Committee. 
Third, the Kaunas Regional Committee for Biomedical Research Ethics, with regard to the epidemiological study protocol (second phase), claimed that all decisions and responsibility on quality and privacy of data is under the Principal Investigator in charge.

This feedback leaves the question open on how to handle and legitimise the shared responsibilities in citizen science projects. This is a significant point considering the nature of citizen science projects in general. The CitieS-Health project represents a good example of attempting to develop procedures which move towards a co-responsibility of researchers and citizen scientists. As a consequence, two key challenges arise that require a more in-depth investigation. First, how to handle the current lack of legal recognition of the involvement of citizens as scientists. ${ }^{6}$ This challenge is also relevant for the concept of participatory governance in the CitieS-Health project, which was designed based on existing literature, in particular, the proposal of the "Every Participant is a Principal Investigator" (EPPI) illustrated in Buyx et al. [2017]. It is evident that the highlighted legal vacuum poses many challenges to experimenting the abstract idea of participatory governance with complete strategies in the field, as Cities-Health is trying to do. Another challenge is how to handle the diversity among European countries concerning the competent Ethics Committees, including the absence, in some of them, of a Research Ethics Committee at University level. As already emphasised, this non-uniformed framework raises particular challenges in addressing the first step of the ethical approval process as well as identifying common guidelines and practices in this regard.

Participatory research designs require renewed attention paid to ethical approval procedures to accommodate the new role of citizens, who are not merely research subjects but provide an active contribution to the research endeavour. In this regard, this paper attempts to shed light on the new elements that should be incorporated into the study protocol and related documents to be submitted to the competent Ethics Committees. Moreover, participatory designs also require paying attention to the challenges that the ongoing collaborative process of citizen science research poses to the traditional process of ethical approval. All these innovations pose some challenges to the current procedure for ethical approval, as highlighted above in reporting the Ethics Committees' feedback.

Another key element regards the central role of public debates with citizens. Public meetings and events are essential in all phases of the study to produce a co-designed protocol; to implement the study in a participatory way; and to devise

\footnotetext{
${ }^{6}$ Note that this challenge also concerns protocols designed, decided, and implemented only by citizens within the so-called participant-led research (PLR). In this context, the reflection has shifted from the initial concern "on whether adequate ethical oversight of PLR must involve standard ethics review" [Vayena and Tasioulas, 2013] to "whether PLR should also be subjected to identical formal oversight mechanisms" [Vayena, Brownsword et al., 2016, p. 217]. In this regard, it was suggested that "[a]lthough ethical and scientific standards apply uniformly across all types of research, the most appropriate means for securing them may vary from one kind of research to another. We should not assume that a one-size-fits-all mechanism of oversight is best. Instead, we must explore various approaches which take into account the distinctive nature of the research in question. Moreover, given the heterogeneous character of PLR activity, different oversight mechanisms may apply to different kinds of research within that general category." [Vayena, Brownsword et al., 2016, pp. 217-218].
} 
a participatory governance. For example, public debates may make it easier to obtain a balance between the contributions of citizens and the researchers. This was the strategy adopted by CitieS-Health researchers for instance on writing protocols and related documents. These materials were prepared by researchers, taking into account the citizens' research questions, inputs and preferences which emerged and were debated during public meetings and events. Once drafted, they were (or will be) presented to citizens for feedback and approval.

Finally, the novelty of ethics for citizens as collaborators poses many difficulties for researchers themselves. For instance, given the involvement of non-professional researchers across all phases of the project - i.e. from the problem formulation, through the research design and implementation, up to the dissemination of findings and related actions, - a new challenge is how to handle potential controversies with respect to information asymmetry, research integrity, citizens' and researchers' differing points of view. The CitieS-Health suggestion is that these novelties are addressed taking into consideration and adapting to the specific context of the investigation, as the researchers involved in the project five pilot studies are, in fact, doing.

Acknowledgments This project has received funding from the European Union's Horizon 2020 research and innovation programme under grant agreement No 824484. This publication reflects only the authors' view. The European Commission is not responsible for any use that may be made of the information contained herein.

\section{References}

ALLEA - All European Academies (2017). The European code of conduct for research integrity. Revised edition. Berlin, Germany.

URL: https : / /www . allea .org/wp-content/uploads/2017/05/ALLEA-European -Code-of-Conduct-for-Research-Integrity-2017.pdf (visited on 20th October 2020).

Biggeri, A. (2019). 'Un'etica per ogni pratica scientifica, anche per quella dei cittadini'. [An ethics for every scientific practice, even for citizen science]. Epidemiologia \& Prevenzione 43 (2-3), pp. 123-124. https://doi.org/10.19191/EP19.2-3.P123.047.

Bonney, R., Ballard, H., Jordan, R., McCallie, E., Phillips, T., Shirk, J. and Wilderman, C. C. (2009). Public Participation in Scientific Research: Defining the Field and Assessing Its Potential for Informal Science Education. A CAISE Inquiry Group Report. Washington, D.C., U.S.A.: Center for Advancement of Informal Science Education (CAISE). URL: http://www. informalscience.org/public-p articipation-scientific-research-defining-field-and-assessing-its-p otential-informal-science.

Buyx, A., Del Savio, L., Prainsack, B. and Völzke, H. (2017). 'Every participant is a PI. Citizen science and participatory governance in population studies'. International Journal of Epidemiology 6 (2), pp. 377-384. https://doi.org/10.1093/ije/dyw204.

Council of Europe (1997). Convention for the protection of human rights and dignity of the human being with regard to the application of biology and medicine: convention on human rights and biomedicine. Oviedo, Spain.

URL: https : //rm. coe. int/CoERMPublicCommonSearchServices/DisplayDCTMC ontent?document Id=090000168007 cf98 (visited on 20th October 2020). 
Council of Europe (2005). Additional protocol to the convention on human rights and biomedicine, concerning biomedical research. Strasbourg, France.

URL: https://rm.coe.int/CoERMPublicCommonSearchServices/DisplayDCTMC ontent?documentId=090000168008371a (visited on 20th October 2020).

- (2016). Recommendation CM/Rec(2016)6 of the Committee of Ministers to Member States on research on biological materials of human origin. URL: https://search.co e. int/cm/Pages/result_details . aspx?ObjectId $=090000168064$ e8ff (visited on 20th October 2020).

De Marchi, B., Biggeri, A., Cervino, M., Mangia, C., Malavasi, G., Gianicolo, E. A. L. and Vigotti, M. A. (2017). 'A participatory project in environmental epidemiology: lessons from the Manfredonia case study (Italy 2015-2016)'. Public Health Panorama 3 (2), pp. 321-335.

URL: https://www . euro.who.int/__data/assets/pdf_file/0008/341558/12 _CaseStudy_Manfredonia_Italy_ENG.pdf.

Den Broeder, L., Devilee, J., Van Oers, H., Schuit, A. J. and Wagemakers, A. (2018). 'Citizen science for public health'. Health Promotion International 33 (3), pp. 505-514. https://doi.org/10.1093/heapro/daw086.

European Commission (14th November 2018). Ethics and data protection. URL: https://ec.europa.eu/info/sites/info/files/5._h2020_ethics_and _data_protection_0.pdf (visited on 20th October 2020).

European Parliament and the Council (2016). Regulation (EC) 2016/679 of 27 April 2016 on the protection of natural persons with regard to the processing of personal data and on the free movement of such data.

URL: https://op.europa.eu/it/publication-detail/-/publication/3e485e 15-11bd-11e6-ba9a-01aa75ed71a1/language-en (visited on 20th October 2020).

- (2018). Regulation (EU) 2018/1807 of the European Parliament and of the Council of 14 November 2018 on a framework for the free flow of non-personal data in the European Union. URL: https://eur-lex. europa. eu/legal-content/EN/TXT/?u ri=CELEX\%3A32018R1807 (visited on 20th October 2020).

Ficorilli, A. (2019). 'Quale etica per la citizen science?' [What ethics for citizen science?] Epidemiologia \& Prevenzione 43 (2-3), pp. 124-125. https://doi.org/10.19191/EP19.2-3.P124.048.

Funtowicz, S. O. and Ravetz, J. R. (1993). 'Science for the post-normal age'. Futures 25 (7). Republished Commonplace (2020), pp. 739-755. https://doi .org/10.1016/0016-3287(93)90022-L.

- (1997). 'Environmental problems, post-normal science and extended peer communities'. In: Études et recherches sur les systèmes agraires et le développement, pp. 169-175.

URL: https://hal. archives-ouvertes.fr/hal-01231607.

Kocman, D., Novak, R., Ficorilli, A., De Marchi, B., Biggeri, A., Gignac, F., Toran, R., Basagana, X., Balestrini, M. and Maccani, G. (31st October 2019). Compilation of good practices in governance models, in research protocols, in bottom-up impact assessment and in informing policy making and local governments. Dissemination level: public, Horizon 2020 CitieS-Health project, pp. 1-139. URL: https://citieshealth.eu/resources/.

Kosmala, M., Wiggins, A., Swanson, A. and Simmons, B. (2016). 'Assessing data quality in citizen science'. Frontiers in Ecology and the Environment 14 (10), pp. 551-560. https://doi.org/10.1002/fee.1436.

Lukyanenko, R., Parsons, J. and Wiersma, Y. F. (2016). 'Emerging problems of data quality in citizen science'. Conservation Biology 30 (3), pp. 447-449. https://doi.org/10.1111/cobi.12706. 
National Academy of Sciences (2009). On being a scientist. A guide to responsible conduct in research. 3rd ed. Washington, DC, U.S.A.: Committee on the conduct of science, National Academy Press. URL: https: //www . nationalacademies . or g/our-work/on-being-a-scientist-a-guide-to-responsible-conduct-in-r esearch-third-edition\#sectionPublications.

Patrick-Lake, B. and Goldsack, J. C. (2019). 'Mind the gap: the ethics void created by the rise of citizen science in health and biomedical research'. The American Journal of Bioethics 19 (8), pp. 1-2. https://doi.org/10.1080/15265161.2019.1639389.

Rasmussen, L. M. (2019). 'When citizens do science: stories from labs, garages and beyond'. Narrative Inquiry in Bioethics 9 (1), pp. 1-4. https://doi.org/10.1353/nib.2019.0001.

Rasmussen, L. M. and Cooper, C. (2019). 'Citizen science ethics'. Citizen Science: Theory and Practice 4 (1), pp. 1-3. https : //doi .org/10.5334/cstp. 235.

Resnik, D. B. and Shampoo, A. E. (2009). Responsible conduct of research. 2nd ed. New York, NY, U.S.A.: Oxford University Press.

Resnik, D. B. (2019). 'Citizen scientists as human subjects: ethical issues'. Citizen Science: Theory and Practice 4 (1), pp. 1-7. https://doi.org/10.5334/cstp. 150.

Resnik, D. B., Elliott, K. C. and Miller, A. K. (2015). 'A framework for addressing ethical issues in citizen science'. Environmental Science $\mathcal{E}$ Policy 54, pp. 475-481. https://doi.org/10.1016/j.envsci.2015.05.008.

United States Environmental Protection Agency (2019). Handbook for citizen science quality assurance and documentation. URL: https://www. epa.gov/sites/product ion/files/2019-03/documents/508_csqapphandbook_3_5_19_mmedits.pdf (visited on 20th October 2020).

Vayena, E., Brownsword, R., Edwards, S. J., Greshake, B., Kahn, J. P., Ladher, N., Montgomery, J., O'Connor, D., O'Neill, O., Richards, M. P., Rid, A., Sheehan, M., Wicks, P. and Tasioulas, J. (2016). 'Research led by participants: a new social contract for a new kind of research'. Journal of Medical Ethics 42 (4), pp. 216-219. https://doi .org/10.1136/medethics-2015-102663.

Vayena, E. and Tasioulas, J. (2013). 'Adapting Standards: Ethical Oversight of Participant-Led Health Research'. PLoS Medicine 10 (3), e1001402. https://doi.org/10.1371/journal.pmed.1001402.

Wiggins, A. and Wilbanks, J. (2019). 'The rise of citizen science in health and biomedical research'. The American Journal of Bioethics 19 (8), pp. 3-14. https://doi.org/10.1080/15265161.2019.1619859.

World Medical Association (2013). Declaration of Helsinki: ethical principles for medical research involving human subjects.

URL: https://www.wma.net/policies-post/wma-declaration-of-helsinki-e thical-principles-for-medical-research-involving-human-subjects / (visited on 20th October 2020).

Authors

Antonella Ficorilli is a bioethicist, graduated in Philosophy. She held a Ph.D. in Bioethics at Bologna University in 2009 and a Master's Degree in Practical Ethics and Bioethics at Sapienza University of Rome in 2005 (Italy). She conducted research activities at Oxford University and other universities and scientific institutions. She is currently participating in the Cities-Health project.

E-mail: ficorilliantonella@gmail.com. 
Giovanni Maccani is currently a Senior Researcher at Ideas for Change. He has been Assistant Professor/Lecturer in Management Information Systems at the School of Business, National University of Ireland Maynooth (NUIM). He has achieved a bachelor's and a master's degree in Engineering, both at the Polytechnic University of Milan. He earned a Ph.D. in Information Systems in 2016 at NUIM. E-mail: giovannimaccani@ideasforchange.com.

Mara Balestrini has a Ph.D. in Computer Science and is research lead at Ideas for Change. She is a co-founder of SalusCoop, a Spanish cooperative that seeks to legitimize the right of citizens to control their own data, while facilitating data sharing to accelerate research and innovation in the health sector.

E-mail: marabalestrini@ideasforchange.com.

Annibale Biggeri is Professor of Medical Statistics, Department of Statistics, Computer Science, Applications "G. Parenti" of the University of Florence (Italy), and President of "Epidemiologia e Prevenzione" Social Enterprise. He is involved in several projects relevant both from the scientific and the citizen communities' point of view, involving interaction with local communities - among other Health Impact of the ILVA Steel Plant (Taranto IT); Health Impact Assessment of Air Pollution at Regional Level, Lombardy Region (IT).

E-mail: annibale.biggeri@unifi.it.

Bruna De Marchi is a sociologist currently associated with the Centre for the Study of the Sciences and Humanities (SVT - Senter for vitenskapsteori) in the University of Bergen, Norway (https:/ / www.uib.no/en/user/7458). She also collaborates with the no-profit consultancy Società per l'Epidemiologia e la Prevenzione "Giulio A. Maccacaro", Milan, Italy (http:/ /www.epidemiologiaprevenzione.it/). E-mail: brunademarchi@gmail.com.

Frederique E. M. Froeling is a Ph.D. candidate at the Institute for Risk Assessment Sciences at Utrecht University, in Utrecht, The Netherlands. She has a MSc in Global health from Maastricht University and her research interests include environmental epidemiology (particularly air pollution), (global) health, and citizen science. E-mail: f.e.m.froeling@uu.nl.

Florence Gignac is currently a Ph.D. student in Biomedicine at Pompeu Fabra University and ISGlobal (Spain). Her main research interest centres on air pollution, mental health and citizen science. She completed her bachelor in Geography and Environmental Sciences at McGill University, Canada, in 2015. She has a postgraduate diploma in International Environmental Health from the School of Public Health of University of Montreal (Canada) and a master's degree in Clinical Investigation from ISGlobal and Barcelona University (Spain). E-mail: florence.gignac@isglobal.org.

Regina Grazuleviciene MD, Dr.habil., graduated from Kaunas Institute of Medicine. She is a Senior Scientist, Professor in Environmental epidemiology and risk assessment at Department of Environmental Sciences Vytauto Didziojo University (VDU), Kaunas, Lithuania. She is an expert in exposure assessment, epidemiology, and health risk assessment, has been involved in EC funded projects as a Principal Investigator: HiWATE, ESCAPE, COPHES, PHENOTYPE, HELIX, and in HORIZON2020 - CitieS-Health and ATHLETE. She is author 110 international publications, H-index of 29. E-mail: regina.grazuleviciene@vdu.lt. 
Gerard Hoek is currently an associate professor in Environmental Epidemiology at the Institute for Risk Assessment Sciences at Utrecht University, Utrecht the Netherlands. His research interests include development of exposure assessment methods and environmental epidemiology of diverse environmental stressors, with a focus on air pollution. E-mail: g.hoek@uu.nl.

Tjaša Kanduč currently works at Jožef Stefan Institute at isotope biogeochemistry group. The research is mainly focused on stable isotope studies in environment, e.g. freshwater systems, marine systems, groundwater systems, coalbed gases. In last years she was also involved in citizen science projects, e.g. ICARUS H2020, CitieS-Health H2020, HBM (Human biomonitoring in Slovenia), where she participated in involvement and communication with citizens.

E-mail: tjasa.kanduc@gmail.com. E-mail: tjasa.kanduc@ijs.si.

David Kocman is Head of Informatics group at Department of Environemntal Sciences at Jožef Stefan Institute. His experience is in environmental data analysis, spatial data acquisition and management, participatory sensing, citizens science and testing of Citizens Observatory concepts. E-mail: david.kocman@ijs.si.

Valeria Righi has a Ph.D. in Human-Computer Interaction from Pompeu Fabra University. She is currently a senior researcher at Ideas for Change and she is involved in various European projects in the field of citizen science and data governance. She is co-founder of Salus.coop, a citizen cooperative of health data for science. E-mail: valeriarighi@ideasforchange.com.

Xavier Basagaña has a Ph.D. in Biostatistics and is currently Associate Research Professor at the Barcelona Institute for Global Health (ISGlobal), where he conducts research on the health effects of air pollution and other environmental exposures. $\mathrm{He}$ is the coordinator of the CitieS-Health project.

E-mail: xavier.basagana@isglobal.org.

How to cite

(C) The Author(s). This article is licensed under the terms of the Creative Commons Attribution - NonCommercial - NoDerivativeWorks 4.0 License. ISSN 1824-2049. Published by SISSA Medialab. jcom.sissa.it 